\title{
ЗАКОНОМЕРНОСТИ ИЗМЕНЕНИЙ ПАРАМЕТРОВ ПРОЦЕССОВ ПЕРЕНОСА МНОГОКОМПОНЕНТНЫХ УГЛЕВОДОРОДНЫХ ГАЗОВЫХ СРЕД В УСЛОВИЯХ СЕПАРАЦИИ
}

\author{
Николаев Евгений Владимирович', \\ nickolaev_evgeny@mail.ru \\ Харламов Сергей Николаевич', \\ kharsn@mail.ru
'Национальный исследовательский Томский политехнический университет, Россия, 634050, г. Томск, пр. Ленина, 30.

При решении прикладных задач по установлению особенностей изменений параметров в газообразных сплошных средах при конвективном тепло- и массообмене, сопротивлении трения в аппаратах и устройствах, обеспечивающих функционирование технологических процессов в нефтегазовой отрасли, ценным является представление о механизмах и интенсивности процессов переноса тепла, массы и импульса. Такие задачи нетривиальны из-за выраженной нелинейности эффектов, нестабильности и разнообразия компонентного состава рабочих смесей в аппаратах и требуют большой априорной информации при моделировании, получение которой ограничено спецификой процессов и их экспериментального анализа. В силу сказанного, исследования, направленные на уяснение и прогноз закономерностей изменений локальных и интегральных свойств гомогенных и гетерогенных углеводородных систем в аппаратах топливно-энергетического комплекса являются актуальными и ценными для практики.

Цель: выявление особенностей и установление закономерностей эволюции локальных параметров и процессов конвективного тепло- и массообмена в нефтегазовых аппаратах (таких как коэффициенты динамической и кинематической вязкости, тепло- и температуропроводности, диффузионности, а также их безразмерных аналогов - чисел Прандтля, Шмидта и Льюиса), ориентированных на использование в качестве рабочего тела многокомпонентных углеводородных газовых сред в диапазоне термобарических условий, характерных для функционирования оборудования подготовки нефти при сепарации $-T \cong 0-70{ }^{\circ} \mathrm{C}$ и $P \cong 0,1-3,5 \mathrm{M \Pi а}$.

Методы: термодинамическая модель в программном комплексе Aspen HYSYS - уравнение состояния реальных газовых смесей Пенга-Робинсона; положения статистической механики, подходы соответственных состояний, методы Чепмена-Энскога, Голубева, теории подобия и анализа размерностей.

Результаты. В рамках положений равновесной термодинамики выполнено детальное исследование эволюции локальных и интегральных параметров процессов переноса импульса, тепла и массы в углеводородных газовых смесях в условиях сепарации при заданных температурах и давлениях. Установлены границы применимости практического использования метода подобия в количественных оценках, и качественных прогнозах механизмов и конфигураций конвективного тепло- и массообмена в устройствах подготовки нефти. Обсуждаются результаты использования метода аналогий и моделирования процесса сепарации в общих задачах процессов переноса импульса, тепла и массы в задачах нефтегазовой отрасли. В практику проектирования оборудования рекомендуются заключения об особенностях изменений свойств в сложных по структуре смесях и интенсивности механизмов тепло- и массообмена при сепарации, нарушающих тройную аналогию в неизотермических гомогенных и гетерогенных средах.

\section{Ключевые слова:}

Коэффициенты переноса, многокомпонентная система, углеводороды, сепарация, моделирование.

\section{Введение}

В настоящее время проектирование, разработка и оптимизация работы промышленных аппаратов не обходится без численного моделирования многомерных и многофакторных процессов в конвективном и диффузионном тепломассопереносе, гидрогазодинамике, осложненных фазовыми переходами, весьма чувствительными к изменению режима течений и структуры теплоносителей, интенсивности механизмов переноса скаляра (тепла, массы) и импульса, а также особенностям распределений теплофизических свойств в сопряженной термодинамической системе «внешняя среда - поверхность промышленных устройств - рабочее тело» [1]. Это связано с прогрессом в исследовании инженерных задач нефтегазовой отрасли в рамках своих общих и полных постановок, опирающихся на привлечение законов сохранения массы индивидуальных компонентов (фаз) в смесях, энергии и импульса, а также в результате появления быстродействующих ПЭВМ и целого ряда программных продуктов, предназначенных для прогноза физико-химических явлений и механизмов в рабочих системах и технологических процессах. Ввиду этого к точности исследования тепло- и массообмена в сплошных гомогенных и гетерогенных системах предъявляются все более высокие требования, в частности, к методикам формулировок краевых условий для математических моделей в многокомпонентных газовых смесях и методам отыскания их решений. Для успешного численного интегрирования нелинейных дифференциальных уравнений и их замыкающих соотношений требуются корректные сведения о деталях поведения теплофизических свойств термодинамической системы (параметрах динамической, кинематической и объемной вязкостях, тепло- и температуропроводности), характеризующих интенсивности протекания процессов 
переноса импульса, теплоты и массы [2]. Следует отметить, что ввиду широкого многообразия рабочих смесей газов в аппаратах экспериментальный подход в решении указанных задач сопряжен со значительными сложностями. И данные теоретического анализа закономерностей и эволюции пространственных распределений локальных и интегральных параметров функционирующих объектов, аппаратов и устройств нефтегазовой отрасли обретают чрезвычайную важность для практики.

Известно, что при определении коэффициентов переноса химически однородных веществ используются два подхода: феноменологический (в рамках принципа соответственных состояний) и статистический (идеи молекулярно-кинетической теории газов). Некоторые результаты сопоставления данных подходов при вычислении коэффициентов динамической вязкости и тепло- и температуропроводности индивидуальных веществ, на примере которых можно увидеть, что они выдают близкие между собой значения и находятся в хорошем согласии с экспериментом, были отражены в [1-3]. Вместе с тем в [3] продемонстрировано, что картина изменений указанных свойств многокомпонентных газовых смесей, полученных с помощью общепринятых методов Уилки [4] и Мэйсона-Саксены [5], качественно коррелирует с результатами, полученными в среде HYSYS. Необходимо отметить, что в отличие от коэффициентов динамической вязкости и теплопроводности вычисление коэффициента диффузии в многокомпонентных смесях требует специального подхода к каждому случаю. Поэтому в последнее время проводится множество исследований, направленных на разработку новых методов определения коэффициента (эффективного, бинарного) диффузии в газах [6-8]. Так, например, в [7, 8] проведены экспериментальные исследования диффузии в бинарных газовых смесях, в широком диапазоне изменений температуры и давления. Также в $[6,7]$ предлагаются новые методы определения коэффициентов бинарной и эффективной диффузии в смесях. Это показывает, что исследования диффузионного переноса массы в смесях далеки от завершения, в особенности для многокомпонентных систем. В практике известны два метода определения эффективного коэффициента диффузии в многокомпонентных гомогенных и гетерогенных газовых смесях - бинарная постановка задачи [9] и метод Уилки [10]. При бинарной постановке смесь разделяется на два псевдокомпонента по близким значениям молекулярной массы, после чего рассчитывается коэффициент бинарной диффузии по известным методам. В случае с методом Уилки эффективный коэффициент диффузии вычисляется для каждой отдельной компоненты смеси. Однако в случаях смесей с сильно различающимися по молекулярной массе компонентами использование обоих методов приводит к большим расхождениям с экспериментом. Поэтому оценка скорости диффузии в газах и по- иск новых методов её определения является актуальным направлением исследования.

Учитывая сказанное, целью данной работы является выявление закономерностей параметров процессов переноса (коэффициентов динамической вязкости, теплопроводности, диффузии, чисел Прандтля, Шмидта и Льюиса) многокомпонентных углеводородных газовых сред в зависимости от термобарических условий сепарации, характерных для функционирования оборудования подготовки нефти $-T \cong 0-70{ }^{\circ} \mathrm{C}$ и $P \cong 0,1-3,5$ МПа.

\section{Детали численного моделирования}

При решении тепло- и массообменных задач в сплошных средах широко используется метод подобия [11]. Анализ изучаемых процессов методом подобия подразумевает приведение уравнений, описывающих исследуемый процесс, и соответствующих краевых (начальных и граничных) условий к безразмерному виду [1-3]. При этом число новых безразмерных переменных и постоянных величин, входящих в основные уравнения и краевые условия, оказывается меньше числа размерных величин, что значительно упрощает задачу. Так, при решении задач теплообмена искомой величиной является безразмерный коэффициент теплоотдачи - число Нуссельта $(\mathrm{Nu})$, массообмена - безразмерный коэффициент массоотдачи - число Шервуда $(S c)$. B простейшей конфигурации конвективного теплои массообмена с достаточной для практики точностью указанные параметры определяются эмпирическими критериальными связями типа [11]:

$$
\begin{aligned}
& N u=f_{1}(\operatorname{Re}, \operatorname{Pr}), \\
& S h=f_{2}(\operatorname{Re}, S c) .
\end{aligned}
$$

Здесь $f_{1}, f_{2}$ - некоторые известные функции, учитывающие физические особенности среды к интенсивности тепломассопереноса под действием внешних и внутренних сил в термодинамических системax; $R e$ - число Рейнольдса, $P r$ - число Прандтля, $S c$ - число Шмидта. Числа Прандтля, Шмидта и Льюиса $(L e=P r / S c)$ являются определяющими критериями подобия. Они определяют тройную аналогию процессов переноса в газах, характеризуя интенсивности между переносами теплоты, массы и импульса. В инженерной практике прикладных расчетов тепло- и массообмена в газах зачастую указанные числа принимают одинаковыми (idem), что позволяет экстраполировать результаты вычислений одного процесса на другие [12]. Однако ни численно, ни экспериментально это не было подтверждено для многокомпонентных газовых смесей. В частности, в [3] показано, что данные числа подобия могут изменяться в достаточно широком диапазоне значений. Следовательно, уяснение характера распределенийперечисленных параметров для многокомпонентных газовых сред является немаловажной задачей. Таким образом, численное исследование зависимостей коэффициентов переноса и чисел подобия многокомпонентных углеводородных сред от 
температуры и давления с учётом изменения компонентного состава в термобарических условиях процесса сепарации, характерных для аппаратов подготовки нефти, проводилось в несколько этапов:

- моделирование процессов сепарации пластовых нефтей различных месторождений в указанном диапазоне изменений температур и давлений;

- расчет коэффициентов динамической вязкости, теплопроводности углеводородных газовых смесей, полученных в результате моделирования сепарации пластовых нефтей;

- выявление зависимости коэффициента самодиффузии веществ от давления, предложение поправочного коэффициента;

- вычисление эффективного коэффициента диффузии в углеводородных средах, а также чисел подобия (Прандтля $P r$, Шмидта $S c$ и Льюиса $L e$ ). Достоверное прогнозирование процесса сепарации является важной частью исследования, поскольку коэффициенты переноса многокомпонентных газовых смесей сильно зависят от состава. Поэтому моделирование сепарации проводилось в программном пакете HYSYS, с использованием в качестве термодинамической модели уравнения состояния Пенга-Робинсона [13, 14]. Ранее в [3] проводили сравнительный анализ некоторых имеющихся моделей пакета HYSYS в прогнозе покомпонентного разделения пластовой нефти, в результате чего подтвердили, что уравнение Пенга-Робинсона действительно выдаёт надежные результаты относительно остальных моделей. Также заметим, что в [15] были получены картины изменений чисел подобия $(P r, S c, L e)$ для двух вариантов газовых смесей для термобарических условий сепарации и высказаны рекомендации по использованию уравнения из кинетической теории газов [16], выражающее коэффициент самодиффузии вещества через коэффициент кинематической вязкости и соотношения интегралов столкновений для переноса импульса и массы, для определения осредненной скорости диффузии в смесях:

$$
D_{i i}=v_{i} \frac{6}{5} \frac{\Omega_{V}}{\Omega_{D}},
$$

где $D_{i i}$ - коэффициент самодиффузии вещества, $\Omega_{V}, \Omega_{D}$ - интегралы столкновений для переноса импульса и массы, соотношение которых для газов при умеренных давлениях приблизительно равно 1,1 . Однако в (3) не было учтено влияние давления на соотношение $\Omega_{V} / \Omega_{D}$, так как оно является функцией характеристической температуры. Поэтому в данной работе предлагается поправочное уравнение, учитывающее давление, вида:

$$
\frac{\Omega_{V}}{\Omega_{D}}=1,0665-0,0551 P \text {. }
$$

Здесь $P$ - давление, МПа. В данном случае число Шмидта не зависит от температуры, что вполне оправдано для чистых веществ и бинарных смесей газов, и с учетом (4) равно:

$$
\text { Sc }=\frac{5}{6}(1,0665-0,0551 P) \text {. }
$$

Таким образом, при известных данных о коэффициентах динамической вязкости, плотности (коэффициентах кинематической вязкости) и числе Шмидта многокомпонентных газовых смесей при определенных термобарических условиях можем найти их эффективные коэффициенты диффузии. В целях верификации представленного метода сопоставлены результаты расчетов с экспериментальными данными для чистых веществ и бинарных смесей (рис. 1, 2).

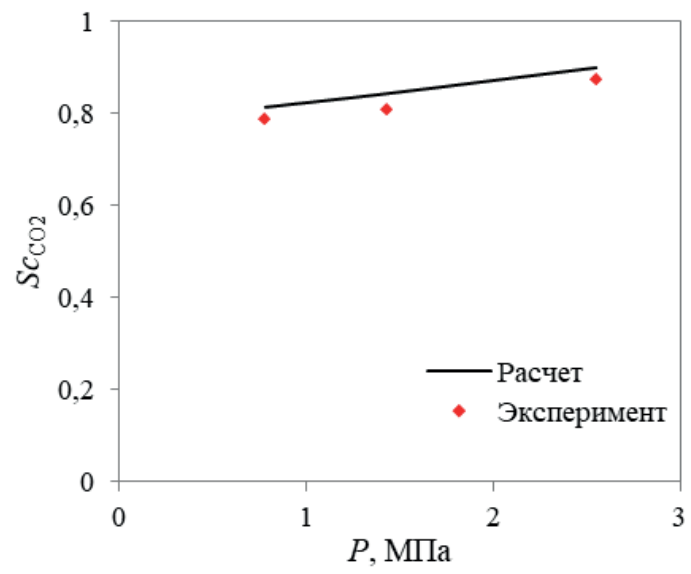

Pис. 1. Экспериментальные и расчетные значения числа Шлидта двуокиси углерода в зависимости от давления

Fig. 1. Experimental and calculated values of the carbon dioxide Schmidt number as a function of pressure

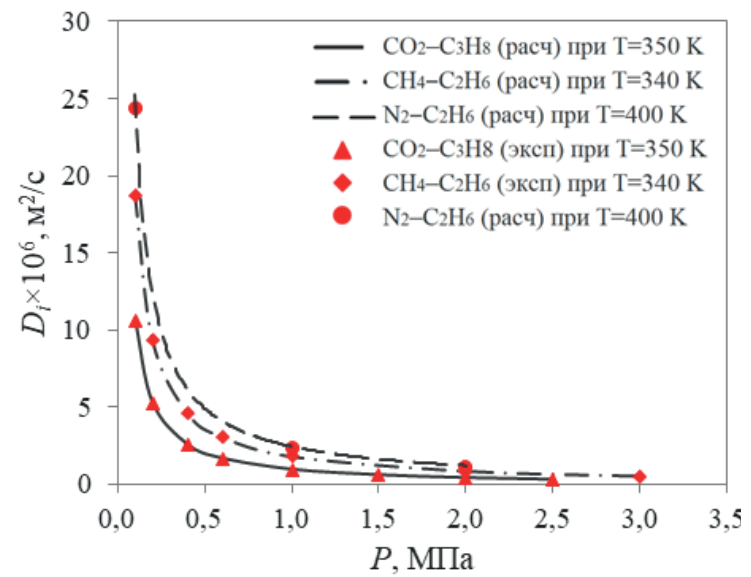

Pис. 2. Экспериментальные и расчетные значения эффективного коэффиииента диффузии бинарных смесей в зависимости от давления при различных температурах

Fig. 2. Experimental and calculated values of binary mixtures effective diffusion coefficient as a function of pressure at various temperatures

На рис. 1, 2 экспериментальная информация была позаимствована из $[17,18]$, при этом средняя относительная погрешность расчетных величин не превышает $4 \%$. Это позволяет утверждать, что предлагаемые уравнения (4) и (5) являются вполне удовлетворительными в прогнозировании эффективного коэффициента диффузии и числа Шмидта в смесях.

\section{Результаты и их обсуждение}

В качестве исходных данных при численном моделировании процессов сепарации углеводород- 


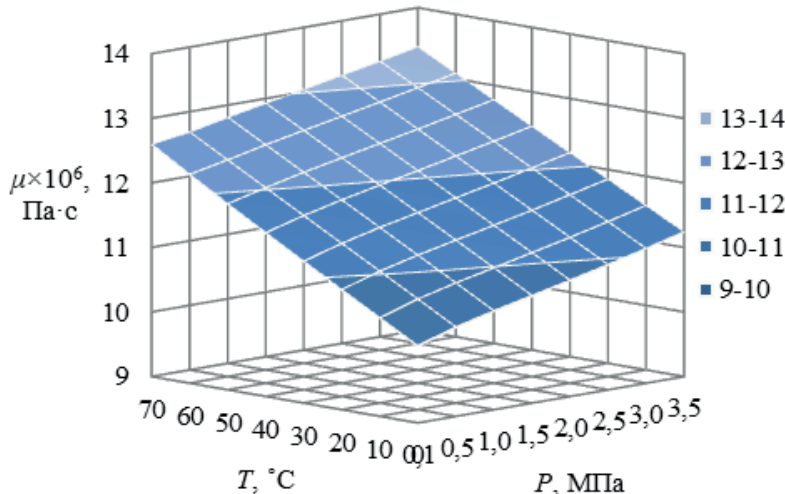

$a / a$

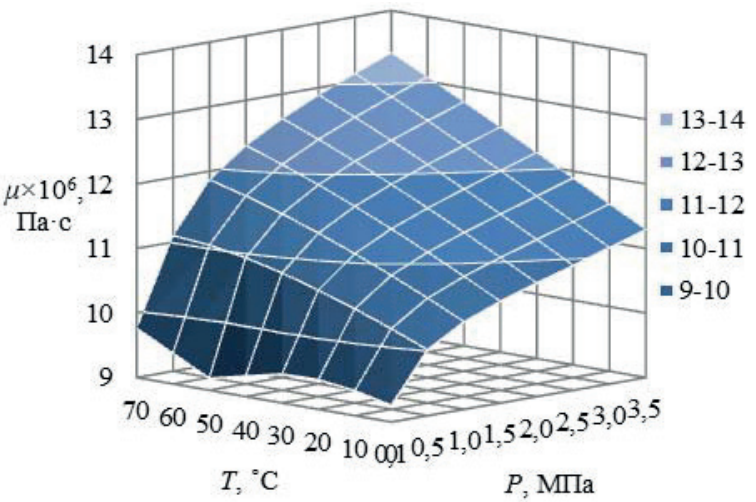

$\sigma / b$

Рис. 3. Эволюиия коэффициента диналической вязкости газовой слеси в зависилости от температуры и давления сепарации пластовой нефти: а) Губкинского месторождения; б) Вынгапуровского месторождения

Fig. 3. Evolution of the gas mixture dynamic viscosity coefficient depending on the temperature and pressure of crude oil separation: a) Gubkinskoe field; b) Vyngapurovskoe field

ных сред были выбраны компонентные составы пластовых нефтей 10 различных месторождений. Заметим, что содержание в смесях основного составляющего - метана - находится в пределах от 27,8 до 98,0 \% (об.). В связи с этим теплофизические свойства у каждой смеси в зависимости от температуры и давления изменяются нетривиально (например, рис. 3).

Однако, в отличие от свойств, числа подобия $(\mathrm{Pr}, \mathrm{Sc}, \mathrm{Le})$ распределяются во всех случаях в достаточно узком диапазоне значений. Так, число Прандтля ограничивается нижним пределом 0,74 и верхним - 0,86, число Шмидта варьируется в пределах величин от 0,79 до 0,95, а число Льюиса - от 1,00 до 1,24 (рис. 4).

Вышеизложенное позволяет утверждать, что в многокомпонентных углеводородных газовых смесях при сепарации в условиях атмосферного давления и температур от 0 до $70{ }^{\circ} \mathrm{C}$ существует подобие процессов переноса теплоты и массы. Следователь-

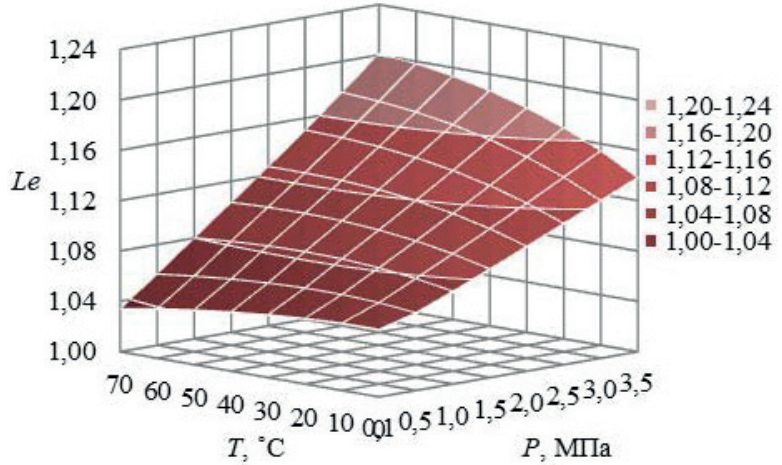

$a / a$ но, функции в выражениях (1) и (2) принимают одинаковый вид, в связи с чем результаты расчета теплообмена могут быть применены для расчета массообмена. Кроме того, при сепарации в условиях давления 3,5 МПа имеем подобие процессов переноса импульса и массы, так как число Шмидта при указанном давлении находится ближе всего к единице $(S c=0,95)$. В этом случае справедливо будет выразить число Шервуда как функцию одной переменной - числа Рейнольдса:

$$
S h=f(R e) .
$$

Таким образом, проведенное исследование параметров процессов переноса в углеводородных газовых смесях в условиях сепарации в пределах рассматриваемых температур и давлений позволило обозначить границы применимости метода подобия, упрощенной критериальной связи (6) и оценить степени отклонения безразмерных параметров, характеризующих аналогию процессов переноса, от единицы. Данные рекомендации полезны

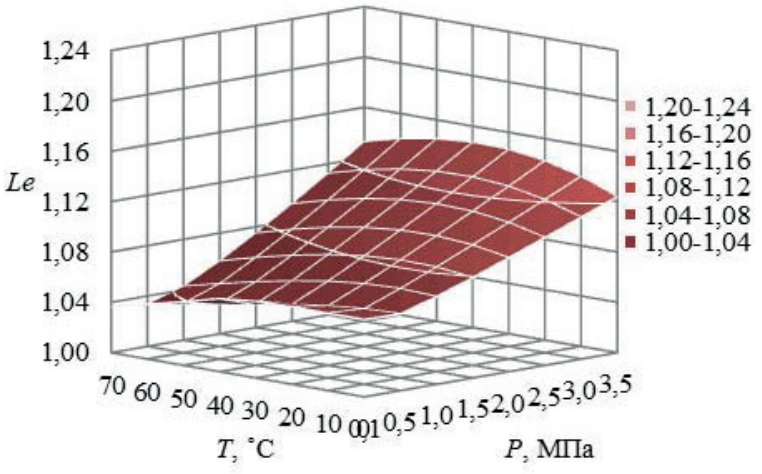

$\sigma / b$

Pис.4. Эволюиия числа Льюиса газовой смеси в зависимости от температуры и давления сепаращии пластовой нефти: а) Губкинского месторождения; б) Вынгапуровского месторождения

Fig. 4. Evolution of the gas mixture Lewis number depending on the temperature and pressure of crude oil separation: a) Gubkinskoe field; $b$ ) Vyngapurovskoe field 
при расчетах тепло- и массообмена в сплошных средах не только в нефтегазовой промышленности, но и в химической, топливно-энергетической и т. д. Об этом свидетельствует использование критериальных связей типа (1) и (2) в самых различных инженерных расчетах: при оценке испарений нефти из резервуаров [19], при моделировании массопереноса в газовой фазе в скруббере Вентури [20], в практике научного приборостроения [12], а также в направлении прикладного моделирования процессов горения [21]. Следует отметить, что до сих пор исследования характера массообмена в углеводородных средах далеки от завершения, в особенности при повышенных давлениях, о чём говорят современные работы [22, 23].

\section{Заключение}

Результаты проведенного исследования позволяют сформулировать следующие выводы.

1. Исследованы зависимости теплофизических свойств (коэффициентов динамической вязкости, теплопроводности и диффузии) углеводо-

\section{СПИСОК ЛИТЕРАТУРЫ}

1. Prospects of RANS Models With Multiparameter Effects at Simulation of Complex Non-isothermal Flows of Viscous Media in Devices With Any Configuration of Surface / S.N. Kharlamov, V.Yu. Kim, S.I. Silvestrov, R.A. Alginov, S.A. Pavlov // Proceedings of the $6^{\text {th }}$ International Forum on Strategic Technology. Harbin, China, August 22-24, 2011. -- V. 2. - P. 787-791.

2. 0 проблемах математического моделирования процессов переноса импульса, тепла и массы в углеводородных вязких средах в условиях сложного движения и тепломассопереноса в трубопроводах / С.Н. Харламов, С.И. Сильвестров, В.В. Зайковский, Е.В. Николаев // Вестник Российской Академии естественных наук. Западно-Сибирское отделение. - 2017. Вып. 20. - С. 67-89.

3. Николаев Е.В., Харламов С.Н. Исследование сепарационных процессов углеводородных многокомпонентных систем в режимах функционирования оборудования предварительной подготовки нефти // Известия Томского политехнического университета. Инжиниринг георесурсов. - 2016. - Т. 327. № 7. - C. 84-89.

4. Wilke C.R. A Viscosity Equation for Gas Mixtures // The Journal of Chemical Physics. - 1950. - V. 18. - P. 517-519.

5. Mason E.A., Saxena S.C. Approximate Formula for the Thermal Conductivity of Gas Mixtures // The Physics of Fluids. - 1958. V. 1. - P. 361-369.

6. Lunev V.V. New phenomenological model of multicomponent gas diffusion // Fluid Dynamics. - 2017. - V. 52. - P. 454-462.

7. Незовитина М.А. Исследование зависимости коэффициентов взаимной диффузии углеводородных газов от давления при различных температурах: дис. ... канд. техн. наук. - Смоленск, 2011. $-189 \mathrm{c}$

8. Zangi P., Rausch M.H., Fröba A.P. Binary Diffusion Coefficients for Gas Mixtures of Propane with Methane and Carbon Dioxide Measured in a Loschmidt Cell Combined with Holographic Interferometry // International Journal of Thermophysics. - 2019. V. 40:18. - P. 1-17.

9. Тирский Г.А. Определение эффективных коэффициентов диффузии в ламинарном многокомпонентном пограничном слое // Доклады академии наук. - 1964. - Т. 155. - №6. C. $1278-1281$. родных газовых смесей от термобарических условий с учётом изменения компонентного состава при сепарации.

2. Предложен метод определения эффективного коэффициента диффузии в многокомпонентных газовых смесях с помощью соотношения из кинетической теории газов для вычисления коэффициента самодиффузии веществ через их плотность и коэффициент кинематической вязкости.

3. Установлено влияние давления на соотношение интегралов столкновений для переноса массы и импульса, и предложено поправочное уравнение для учёта параметра давления.

4. Впервые приведены и обобщены числа Прандтля $P r \cong 0,74 \ldots 0,86$, Шмидта $S c \cong 0,79 \ldots 0,95$ и Льюиса $L e \cong 1,00 \ldots 1,23$ для многокомпонентных углеводородных газовых сред в условиях сепарации пластовой нефти различных месторождений, определяющие характер тепло- и массообмена в открытых термодинамических системах.

10. Wilke C.R. Diffusional properties of multicomponent gases // Chemical Engineering Progress. - 1950 - V. 46. - P. 95-104.

11. Лыков А.В., Михайлов Ю.А. Теория тепло- и массопереноса. М.: Энергоиздат, 1963. - 563 с.

12. Шарфарец Б.П. Обзор теории явлений переноса и поверхностных явлений применительно к решению некоторых задач научного приборостроения // Научное приборостроение. 2015. - T. 25. - № 3. - C. 45-64.

13. HYSYS 2004 Simulation Basis // URL: https://sites.ualberta.ca/CMENG/che312/F06ChE416/HysysDocs/AspenHYSYSSimulationBasis.pdf (дата обращения 07.07.2019).

14. Peng D.Y., Robinson D.B. A new two-constant equation of state // Industrial \& Engineering Chemistry Fundamentals. - 1976. V. $15 .-$ P. 59-64.

15. Николаев Е.В., Харламов С.Н. Особенности сепарации многокомпонентных углеводородных сред в режимах работы аппаратов подготовки нефти // Известия Томского политехнического университета. Инжиниринг георесурсов. - 2018. T. 329. - № 3. - C. 69-76.

16. Hirshfelder J.O., Curtis C.F., Bird R.B. Molecular Theory of Gases and Liquids. - New York: John Wiley and Sons, 1954. $1219 \mathrm{p}$.

17. Варгафтик Н.Б. Справочник по теплофизическим свойствам газов и жидкостей. - М.: Наука, 1972. - 720 с.

18. Новый справочник химика и технолога. Электродные процессы. Химическая кинетика и диффузия. Коллоидная химия / Р.Ш. Абиев, Е.Е. Бибик, Е.А. Власов, Б.С. Ермаков. - С.-Пб.: Автономная некоммерческая организация, научно-просветительская организация «Профессионал», 2004. -838 с.

19. Любин Е.А., Коршак А.А. Критериальные уравнения массоотдачи при операциях с нефтями в вертикальных цилиндрических резервуарах // Электронный журнал «Нефтегазовое дело». - 2010. - № 2. URL: http://ogbus.ru/files/ogbus/authors/ Lyubin/Lyubin_1.pdf (дата обращения 02.04.2019).

20. Iodine removal effeciency in non-submerged and submerged selfprimming venturi scrubber / M. Ali, Ch. Yan, Zh. Sun, H. Gu, J. Wang, M. Khurram // Nuclear engineering and technology. 2013. - V. 45. - № 45. - P. 203-210.

21. Effect of heat and mass transfer on the combustion stability in catalytic micro-combustors / J. Chen, L. Yan, W. Song, D. Xu // Applied Thermal Engineering. - 2018. - V. 131. - P. 750-765. 
22. Carreon-Calderon B., Uribe-Vargas V. Thermomechanical Point of View of the Effect of Pressure and Free Volume on the Molecular Diffusion Coefficients // Journal of Chemical and Engineering Data. - 2019. - V. 64. - P. 1956-1969.

23. Absorption and desorption mass transfer rates as a function of pressure and mixing in a simple hydrocarbon system / A.B. Dani-

\section{Информация об авторах}

Николаев E.B., аспирант отделения нефтегазового дела Инженерной школы природных ресурсов Национального исследовательского Томского политехнического университета.

Харламов С.Н., доктор физико-математических наук, профессор, профессор отделения нефтегазового дела Инженерной школы природных ресурсов Национального исследовательского Томского политехнического университета. el, S.A. Mohammad, M.A. Miranda, Aichele C.P. // Chemical Engineering Research and Design. - 2019. - V. 144. - P. 209-215.

Поступила 28.08.2019 2. 


\title{
REGULARITIES OF CHANGES IN PARAMETERS OF MULTICOMPONENT HYDROCARBON MEDIA TRANSFER IN SEPARATION CONDITIONS
}

\author{
Eugeniy V. Nikolaev', \\ nickolaev_evgeny@mail.ru \\ Sergey N. Kharlamov', \\ kharsn@mail.ru
'National Research Tomsk Polytechnic University, 30, Lenin Avenue, Tomsk, 634050, Russia.

In solving applied tasks of determining the characteristics of parameters changes in gaseous continuous media during convective heat and mass transfer, friction resistance in devices and equipment ensuring the operation of technological processes in the oil and gas industry, the understanding of mechanisms and intensity of heat, mass and momentum transfer processes is valuable. Such tasks are nontrivial because of the pronounced nonlinearity of effects, instability and diversity of the composition of mixtures, as the working medium of the apparatus, and require large a priori information in modeling, the obtaining of which is limited by specifics of the processes and their experimental analysis. The studies aimed at clarifying and predicting the patterns of changes in the local and integral properties of homogeneous and heterogeneous hydrocarbon systems in the apparatuses of fuel and energy complex are relevant and valuable for practice.

The main aim of the study is to identify features and establish patterns of local parameters evolution of convective heat and mass transfer in oil and gas devices (such as the coefficients of dynamic and kinematic viscosity, heat and thermal diffusivity, diffusion, and their dimensionless analogs - Prandtl, Schmidt and Lewis numbers), focused on the use as working fluid of multicomponent hydrocarbon gas media in the range of temperature and pressure conditions typical for operation of oil preparation equipment at separation $-T \cong 0-70^{\circ} \mathrm{C}$ and $P \cong 0,1-3,5 \mathrm{MPa}$.

The methods: thermodynamic model in HYSYS software - Peng-Robinson equation of state, statistical mechanics, the principle of corresponding states, the Chapman-Enskog, Golubev methods, similarity theory and dimension analysis.

Results. Within the framework of equilibrium thermodynamics the authors have carried out the detailed study of the evolution of local and integral parameters of momentum, heat and mass transfer in hydrocarbon gas mixtures under separation conditions at given temperatures and pressures in the working media. The limits of applicability of the practical use of the similarity method in quantitative estimates and qualitative predictions of the mechanisms and configurations of convective heat and mass transfer in oil preparation devices are established. The results of using the method of analogies in modeling separation in general issues of the momentum, heat and mass transfer in the problems of the oil and gas industry are discussed. The authors recommend in practice of designing equipment the conclusions on the peculiarities of properties changes in mixtures that are complex in structure and intensity of heat and mass transfer mechanisms during separation, violating the triple analogy in non-isothermal homogeneous and heterogeneous media.

\section{Key works}

Transport coefficients, multicomponent system, hydrocarbons, separation, modeling

\section{REFERENCES}

1. Kharlamov S.N., Kim V.Yu., Silvestrov S.I., Alginov R.A., Pavlov S.A. Prospects of RANS Models With Multiparameter Effects at Simulation of Complex Non-isothermal Flows of Viscous Media in Devices with Any Configuration of Surface. Proceedings of the 6 th International Forum on Strategic Technology. Harbin, China, August 22-24, 2011. Vol. 2, pp. 787-791.

2. Kharlamov S.N., Silvestrov S.I., Zaykovskiy V.V., Nikolaev E.V. 0 problemakh matematicheskogo modelirovaniya protsessov perenosa impulsa, tepla i massy v uglevodorodnykh vyazkikh sredakh v usloviyakh slozhnogo dvizheniya i teplomassoperenosa v truboprovodakh [On the problems of mathematical modeling of momentum, heat and mass transfer in viscous hydrocarbon media under conditions of complex motion and heat and mass transfer in pipelines]. Vestnik Rossiykoy Akademii estestvennykh nauk. Zapadno-Sibirskoe otdelenie, 2017, no. 20, pp. 67-89.

3. Nikolayev E.V., Kharlamov S.N. Research of multicomponent hydrocarbon systems separation in modes of functioning of oil preliminary preparation equipment. Bulletin of the Tomsk Polytechnic University. Geo Assets Engineering, 2016, vol. 327, no 7, pp. 84-99. In Rus.

4. Wilke C.R. A Viscosity Equation for Gas Mixtures. The Journal of Chemical Physics, 1950, vol. 18, pp. 517-519.

5. Mason E.A., Saxena S.C. Approximate Formula for the Thermal Conductivity of Gas Mixtures. The Physics of Fluids, 1958, vol. 1, pp. 361-369.
6. Lunev V.V. New phenomenological model of multicomponent gas diffusion. Fluid Dynamics, 2017, vol. 52, pp. 454-462.

7. Nezovitina M.A. Issledovanie zavisimosti koeffitsientov vzaimnoy diffuzii uglevodorodnykh gazov ot davleniya pri razlichnykh temperaturakh. Dis. kand. nauk [Research of dependence of the mutual diffusion coefficients of hydrocarbon gases on pressure at various temperatures. Cand. Diss.]. Smolensk, 2011. 189 p.

8. Zangi P., Rausch M.H., Fröba A.P. Binary Diffusion Coefficients for Gas Mixtures of Propane with Methane and Carbon Dioxide Measured in a Loschmidt Cell Combined with Holographic Interferometry. International Journal of Thermophysics, 2019, vol. 40:18, pp. 1-17.

9. Tirskiy G.A. Opredelenie effektivnykh koeffitsientov diffuzii v laminarnom mnogokomponentnom pogranichnom sloe [Determination of effective diffusion coefficients in a laminar multicomponent boundary layer]. Doklady akademii nauk, 1964, vol. 155, no. 6, pp. 1278-1281.

10. Wilke C.R. Diffusional properties of multicomponent gases. Chemical Engineering Progress, 1950, vol. 46, pp. 95-104.

11. Lykov A.V., Mikhaylov Yu.A. Teoriya teplo- $i$ massoperenosa [Theory of heat and mass transfer]. Moscow, Enegroizdat Publ., $1963.563 \mathrm{p}$.

12. Sharfarec B.P. An Overview of the Theory of Transport Phenomena and Surface Phenomena in Relation to the Solution of Some Problems of Analytical Instrumentation. Nauchnoe priborostroenie, 2015, vol. 25, no. 3, pp. 45-64. In Rus. 
13. HYSYS. 2004. Simulation Basis. Available at: https://sites.ualberta.ca/CMENG/che312/F06ChE416/HysysDocs/AspenHYSYSSimulationBasis.pdf (accessed 07 Jule 2019).

14. Peng D.Y., Robinson D.B. A new two-constant equation of state. Industrial \& Engineering Chemistry Fundamentals, 1976, vol. 15 , pp. 59-64.

15. Nikolayev E.V., Kharlamov S.N. Features of multicomponent hydrocarbon media separation in operating modes of oil preparation apparatus. Bulletin of the Tomsk Polytechnic University. Geo Assets Engineering, 2018, vol. 329, no. 3, pp. 69-76. In Rus.

16. Hirshfelder J.O., Curtis C.F., Bird R.B. Molecular Theory of Gases and Liquids. New York, John Wiley and Sons, 1954. $1219 \mathrm{p}$.

17. Vargaftik N.B. Spravochnik po teplofizicheskim svoystvam gazov $i$ zhidkostey [Directory of thermal and physical properties of gases]. Moscow, Nauka Publ., 1972. 720 p.

18. Abiev P.Sh., Bibik E.E., Vlasov E.A., Ermakov B.S. Novy spra vochnik khimika i tekhnologa. Elektrodnye protsessy. Khimicheskaya kinetika i diffuziya. Kolloidnaya khimiya [New directory of chemist and technologist. Electrode processes. Chemical kinetics and diffusion. Colloid chemistry]. St.-Petersburg, Professional Publ., 2004. 838 p.

19. Lyubin E.A., Korshak A.A. Kriterial'nye uravneniya massootdachi pri operaciyah s neftyami v vertikal'nyh cilindricheskih rezervuarah [Criteria equations of mass transfer during operations with oils in vertical cylindrical tanks]. Oil and Gas Business, 2010, no 2, pp. 1-11. In Rus. Available at: http://ogbus.ru/files/ogbus/authors/Lyubin/Lyubin_1.pdf/ (accessed 02 April 2019).

20. Ali M., Yan Ch., Sun Zh., Gu H., Wang J., Khurram M. Iodine removal effeciency in non-submerged and submerged self-primming venturi scrubber. Nuclear engineering and technology, 2013, vol. 45, no. 45, pp. 203-210.

21. Chen J., Yan L., Song W., Xu D. Effect of heat and mass transfer on the combustion stability in catalytic micro-combustors. Applied Thermal Engineering, 2018, vol. 131, pp. 750-765.

22. Carreon-Calderon B., Uribe-Vargas V. Thermomechanical Point of View of the Effect of Pressure and Free Volume on the Molecular Diffusion Coefficients. Journal of Chemical and Engineering Data, 2019, vol. 64, pp. 1956-1969.

23. Daniel A.B., Mohammad S.A., Miranda M.A., Aichele C.P. Absorption and desorption mass transfer rates as a function of pressure and mixing in a simple hydrocarbon system. Chemical Engineering Research and Design, 2019, vol. 144, pp. 209-215.

Received: 28 August 2019.

\section{Information about the authors}

Eugeniy V. Nikolaev, postgraduate, National Research Tomsk Polytechnic University. Sergey N. Kharlamov, Dr. Sc., professor, National Research Tomsk Polytechnic University. 